\title{
Ship Detection Method in Complex Sea Background
}

\author{
Cuifang Zhao ${ }^{1}$,Mengfan $\mathrm{Che}^{2+}$ \\ 'Zhejiang Normal University,Jinhua Zhejiang,321000,China
}

\begin{abstract}
Absrtact. in view of the influence of sea clutter, scale change of ship and blurred picture caused by environmental factors, this paper proposes a new method of ship detection based on saliency analysis and improved HOG. Firstly, the saliency map is obtained by two-dimensional Gabor multi-directional filtering, and the confidence interval of the multi-directional saliency map is calculated to segment the image, and the segmented image is fused to obtain the final regional proposal. Then, according to the influence of ship scale change and blurry, a feature extraction algorithm based on the combination of Gaussian pyramid and Hog is proposed. Compared with the traditional Hog features, this method has better robustness. Finally, the trained SVM model is used to block by block detection the regional proposal. The experimental results show that the detection accuracy of the algorithm is $93.91 \%$ in many scenes on the sea, which can effectively achieve the detection of ships on the sea.
\end{abstract}

Keywords: ship detection, saliency analysis,SVM classifier.

\section{Introduction}

In recent years, with the rapid development of marine economy, the number of ships and the traffic density in the sea area are increasing. In the face of such arduous tasks as controlling maritime transportation, counting the number of ships and monitoring the number of ships in real time, it is of great significance to study the detection of marine ship targets.

Feature extraction is an important step in ship detection. Wang Huili et al [1] proposed a principal direction weighted hog feature extraction algorithm. Dan Shi et al. [2] extract the local texture of the target with LBP. He Baigen et al [3] proposed a feature extraction algorithm of Canny operator and two parameter CFAR. These feature extraction algorithms do not fully consider the influence of ship target scale changes and fuzzyiness. In the background modeling methods, m Kristan et al. [4] establish the background model through the Gaussian mixture model, and segment the target of the sea surface by the difference subtraction method. Liang Zhao et al. [5] obtain the ship's position through saliency and context information.Yunchuan GUI et al. [6] connect multiple feature maps to obtain multiple scale features for ship detection. Xiaowu Xiao et al [7] proposed a novel deep encoding-decoding framework for ship detection.. These methods are all used in remote sensing images, and do not consider the influence of sea clutter in visible image. There are also methods for ship detection by extracting sea antenna [8] [9] [10], which can only detect ship targets near the sea antenna.

In this paper, a new method of ship detection based on saliency analysis and improved Hog are proposed for complex marine scene. In order to deal with the difficulties brought by sea clutter, ship's scale change and ship's bluzziness, this paper uses multi-directional two-dimensional Gabor transform to get the saliency map, then divides the saliency map to get the region proposal of the targets,Hog feature based on Gauss pyramid for computing potential target, and detects the potential targets by the trained SVM model, The final test results are obtained.

Corresponding author. Tel.: +15605793611.

E-mail address: 310473098@qq.com 


\section{Image saliency analysis}

The sea water area is mainly composed of ships and sea clutter, and the image scene is complex and contains a larger amount of information. For the sea clutter area, the imaging color is balanced, the pixel value is similar, the texture features are not obvious and the gradient direction is not uniform, etc. But for the ship area, the imaging color is prominent, the image edge is obvious, and the gradient direction is relatively uniform, etc. In order to improve the detection performance of ships, it is necessary to find the feature that can effectively distinguish the sea clutter and the ships. In contrast, human visual system is more likely to pay attention to the artificial objects with rich textures and obvious edges. Image saliency feature extraction is a technology that imitates human visual observation process and extracts the region of interest of human eyes. The frequency and direction of Gabor filters are similar to human vision system. So here the Gabor filter is used to extract the saliency information.

The kernel function of two-dimension Gabor transform can be divided into real part and virtual part. The real part is used to smooth the image, further reduce the texture characteristics of sea clutter, and also reduce the impact of light; the virtual part is used for edge detection, highlighting the edge characteristics of ship objects. It also has good robustness to the change of illumination. Five directions $(u=0,45,90,135,180)$ are selected for Gabor filter in each channel of Lab. And in each channel, the Gabor characteristic map is gotten based on the maximum value of Gabor filter results in different directions, listed as following:

$$
M_{c}(x, y)=\max \left(I_{c}(x, y) * g_{u v}(x, y)\right)
$$

Here, $I_{c}(x, y)$ represents one channel of the original image under the Lab model; $g_{u v}(x, y)$ is the Gabor filter template; $u$ is the direction and $v$ is the layer.

Then the saliency map can be calculated as following:

$$
\operatorname{Sal}_{\text {map }}(x, y)=\left\|I_{L}(x, y)-M_{L}(x, y)+I_{a}(x, y)-M_{a}(x, y)+I_{b}(x, y)-M_{b}(x, y)\right\|
$$

Here, $\operatorname{Sal}_{\text {map }}(x, y)$ is the saliency map ; $I_{L}(x, y), I_{a}(x, y)$ and $I_{b}(x, y)$ are the different channel of the original image under Lab model; $M_{L}(x, y), M_{a}(x, y)$ and $M_{b}(x, y)$ are the Gabor characteristic map in different channel.|| || represents Euclidean distance.

\section{Ship Detection}

After saliency analysis, most of the sea clutter background is suppressed and the ship target is prominent. Furthermore, image segmentation can be used to extract potential targets.

Through the segmentation of the original image, the candidate regions of the target are obtained, which need to be extracted to obtain more accurate discrimination, further reduce the false alarm and get more accurate target detection results. Based on the edge information of the ship, this paper improves the traditional hog algorithm, and proposes a feature extraction algorithm (G-Hog) based on the combination of Gaussian pyramid and Hog, which uses the trained SVM classifier to detect the target in the candidate area.

Input original
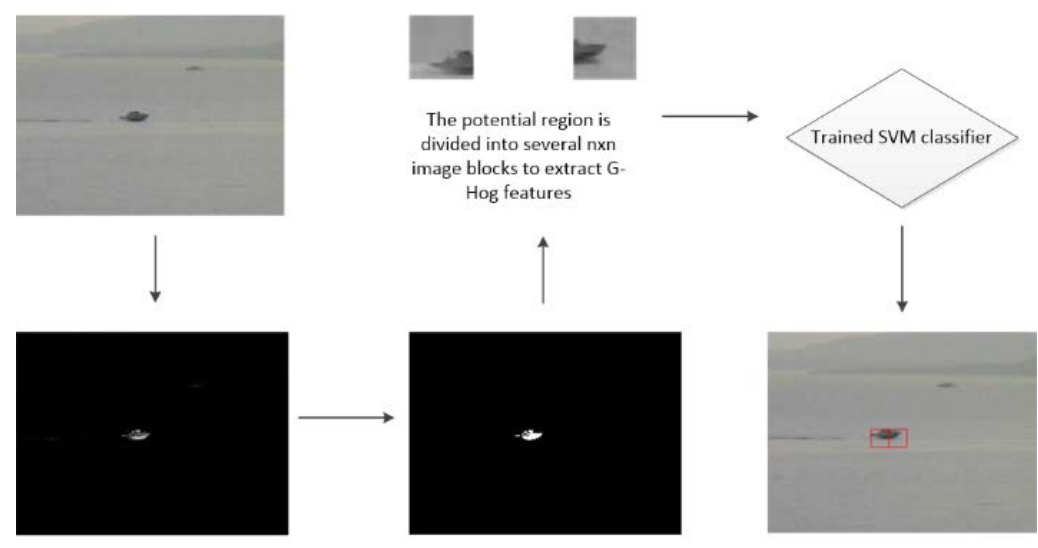

Saliency map

Image segmentation to obtain target candidate

detection result position

Fig.1: The process of ship detection 


\subsection{G-Hog Feature Extraction Algorithm}

Ship targets on the sea often have different sizes and intelligibility. The traditional hog feature extraction algorithm has poor ability to represent these ship features, which makes the detection accuracy low. Different layers of different layers of the Gaussian pyramid have different scales and resolutions. The image size of the bottom layer is larger than that of the top layer, which can reflect the details of the image. With the size and resolution of the image moving up layer by layer is lower, it can reflect the main body information of the object in the image. In this paper, we use the multi-scale representation of the Gaussian pyramid model to construct the Gaussian pyramid for the input detection image, extract the Hog features of each layer of image, and concatenate the hog features of each layer of image as the final feature. This feature not only describes the details of the target, but also describes the main information of the target, which effectively improves the detection accuracy of the target.

Gauss convolution kernel is the only linear kernel to realize scale transformation. For a two-dimensional image, its scale space is defined as:

$$
\begin{aligned}
L(x, y, \sigma) & =G(x, y, \sigma) * I(x, y) \\
G(x, y, \sigma) & =\frac{1}{2 \pi \sigma^{2}} e^{-\left(x^{2}+y^{2}\right) / 2 \sigma^{2}}
\end{aligned}
$$

Here, $I(x, y)$ is the original image; $L(x, y, \sigma)$ represents the image after Gaussian blurring; $G(x, y, \sigma)$ is a Gaussian kernel function.

After the Gaussian pyramid image is established, the local block of potential target is extracted, then ,the descriptor of the local block is gotten by linking the HOG features in each scale.

\subsection{SVM Training and Testing Ship Image}

In this paper, we use the method of block training and testing to divide the original image into small blocks with NxN size, positive samples with target, negative samples with tag 1, negative samples with tag 1. G-Hog of positive and negative samples are extracted and trained in SVM.

The potential regions of the segmented target are mapped back to the original image, and these regions are divided into equal sized NxN blocks and put into the trained model to judge whether there is a target or not.

\section{Experimental results and analysis}

The data set used in this paper is all from the ship video captured by the cruise camera. The data set is divided into single ship part and multi ship part. The ships in these videos are different in type, size and shooting distance. 1500 pictures are randomly selected from the single ship data set as the most training set, and 500 pictures are randomly selected from the multi ship data set as the test set. The size of the pictures is $320 \times 240$. In the experiment, the original image is divided into 32x32 size image blocks, and some results are shown in the Fig.1.
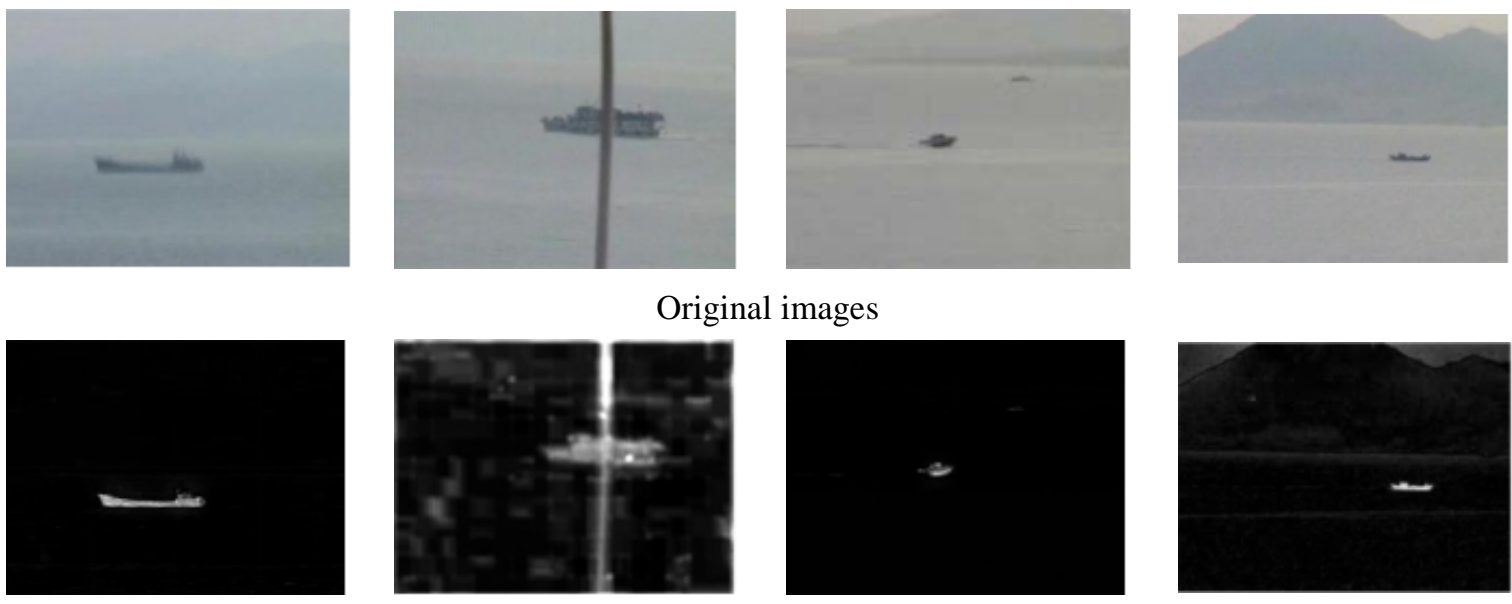

Saliency maps 

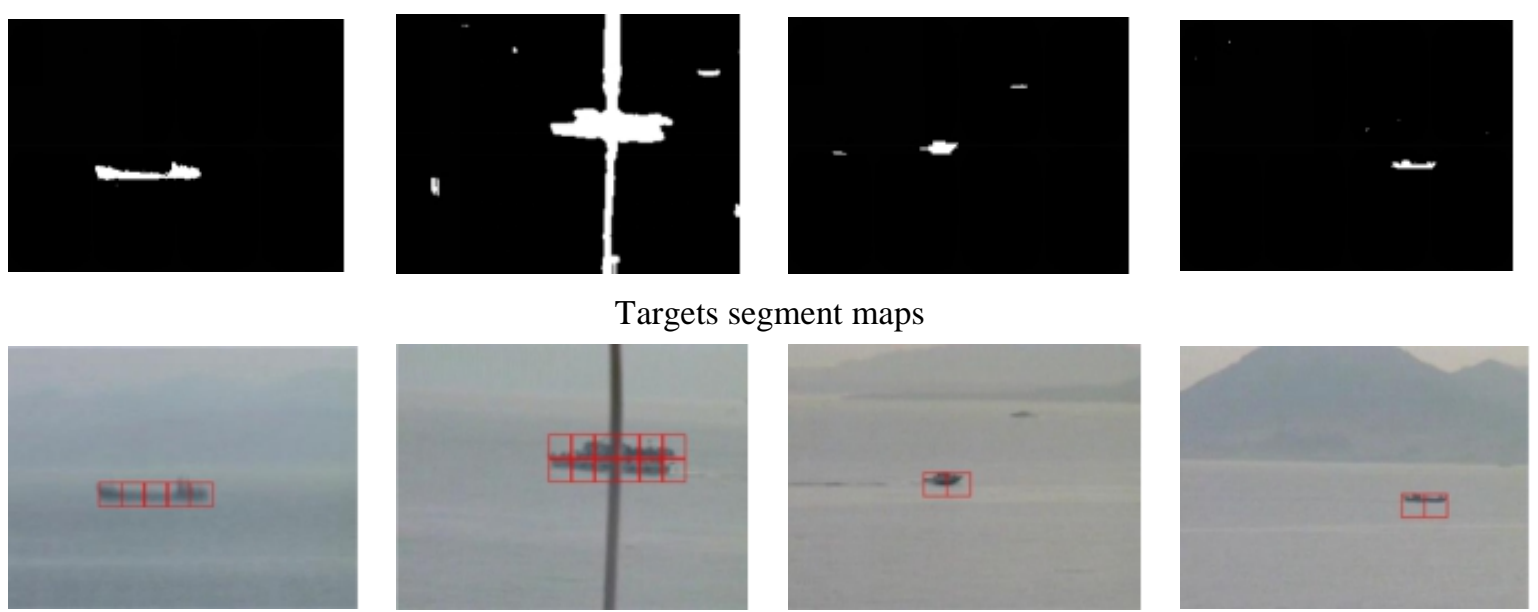

Detection results

Fig.2: Partial results

In order to verify the rationality of dividing the size of 32x32, this paper compares the test results of dividing the size of 32x32 with that of 16x32. When building the Gaussian pyramid, the number of groups is the key to describe the multi-scale image. The more the number of groups is, the more blurred the image is, and the edge is diluted, which can not improve the detection accuracy, but will cause interference to the results. In this paper, the results of two and three groups of Gaussian pyramid are compared. The results are shown in Table 1 and table 2. The method in this paper is compared with hog algorithm and Phog algorithm, and the results are shown in Table 3.

Table 1: Detection results when the number of Gaussian pyramids is 2

\begin{tabular}{ccc}
\hline Size of image block & $16 \times 32$ & $32 \times 32$ \\
\hline detection rate & $77.7 \%$ & $93.91 \%$ \\
mistake rate & $6.39 \%$ & $5.94 \%$ \\
\hline
\end{tabular}

Table 2: Detection results when the number of Gaussian pyramids is 3

\begin{tabular}{ccc}
\hline Size of image block & $16 \times 32$ & $32 \times 32$ \\
\hline detection rate & $78.35 \%$ & $82.7 \%$ \\
mistake rate & $6.65 \%$ & $7.56 \%$
\end{tabular}

From the results in Table 1 and table 2, it can be seen that the number of groups of Gaussian pyramid is not the more the better, which needs to be reasonably established according to the actual situation.In this paper, the number of layers of Gauss pyramid is taken as 2.the detection results of 32x32 small blocks of the image are better than those of 16x32.

Table 3: Algorithm comparison

\begin{tabular}{ccc}
\hline Methods & detection rate & mistake rate \\
& $/ \%$ & $/ \%$ \\
\hline Ours & $93.91 \%$ & $5.94 \%$ \\
HOG & $84.40 \%$ & $7.56 \%$ \\
PHOG & $73.27 \%$ & $5.99 \%$ \\
\hline
\end{tabular}

\section{Conclusion}

In this paper, a new method of visible light ship detection is proposed, which can effectively solve the influence of sea clutter, ship scale change and ship blur in the process of ship detection. The original image is processed by multi-directional two-dimensional Gabor transform, and the saliency map is obtained, and the 
candidate region of the target is obtained by the confidence interval segmentation of the saliency map; then the feature extraction algorithm combined with Gauss pyramid and hog is used to characterize the appearance of the ship, and the candidate region is further detected by parameter training of SVM, and the accurate position of the ship is finally obtained .Experimental results show that the algorithm has good anti-jamming ability

\section{References}

[1] Shi, Dan, et al. "Fusing local texture description of saliency map and enhanced global statistics for ship scene detection." 2015 IEEE International Conference on Progress in Informatics and Computing (PIC). IEEE, 2015.

[2] Yang G , Li B , Ji S , et al. Ship Detection From Optical Satellite Images Based on Sea Surface Analysis[J]. IEEE Geoscience \& Remote Sensing Letters, 2014, 11(3):641-645.

[3] He, Baigen, Xiangying Kong, and Liang Li. "Sea Ship Target Detection Method of Remote Sensing Image." 2019 International Conference on Artificial Intelligence and Advanced Manufacturing (AIAM). IEEE, 2019.

[4] Kristan, Matej, et al. "Fast image-based obstacle detection from unmanned surface vehicles." IEEE transactions on cybernetics 46.3 (2015): 641-654.

[5] Zhai, Liang, Yu Li, and Yi Su. "Inshore ship detection via saliency and context information in high-resolution SAR images." IEEE geoscience and remote sensing letters 13.12 (2016): 1870-1874.

[6] Gui, Yunchuan, et al. "A scale transfer convolution network for small ship detection in SAR images." 2019 IEEE 8th Joint International Information Technology and Artificial Intelligence Conference (ITAIC). IEEE, 2019.

[7] Xiao, Xiaowu, et al. "Accurate Ship Detection via Paired Semantic Segmentation." 2019 Chinese Control And Decision Conference (CCDC). IEEE, 2019.

[8] Ma, Tao, and Jie Ma. "A sea-sky line detection method based on line segment detector and Hough transform." 2016 2nd IEEE International Conference on Computer and Communications (ICCC). IEEE, 2016.

[9] Liu, Qingsong, Xiaofang Xie, and Pengfei Fan. "A Fast Method for Obtaining the Region of Interest of Coastal Infrared Ship." 2016 8th International Conference on Intelligent Human-Machine Systems and Cybernetics (IHMSC). Vol. 2. IEEE, 2016.

[10] Zhang, Haiying, Zhao Zhao, and Fangxiong Xiao. "Robust Detection Method of Small Targets in Sea-Clutter via Improved Fast Clustering Segmentation." 2016 8th International Conference on Intelligent Human-Machine Systems and Cybernetics (IHMSC). Vol. 2. IEEE, 2016. 